\title{
Protein transduction domain-hA20 fusion protein protects endothelial cells against high glucose-induced injury
}

\author{
C.L. Hou ${ }^{1 *}$, Q. Huang ${ }^{2,3 *}$, Y. Wei ${ }^{1}$, W. Zhang ${ }^{1}$, J.H. Mi ${ }^{1}$, D.J. Ying ${ }^{1}$ and \\ Z.H. Zhou ${ }^{4}$
}

${ }^{1}$ Key Lab of Biomechanics, Department of Anatomy, Third Military Medical University, Chongqing, China ${ }^{2}$ Department of Orthopaedics, Southwest Hospital, Third Military Medical University, Chongqing, China ${ }^{3}$ Department of Orthopaedics, Shapingba Traditional Chinese Medicine Hospital, Chongqing, China

${ }^{4}$ Department of Neurology, Southwest Hospital, Third Military Medical University, Chongqing, China

*These authors contributed equally to this study.

Corresponding author: C.L. Hou

E-mail: houchunliwf@163.com

Genet. Mol. Res. 11 (3): 1899-1908 (2012)

Received November 10, 2011

Accepted April 10, 2012

Published July 19, 2012

DOI http://dx.doi.org/10.4238/2012.July.19.9

\begin{abstract}
We constructed a plasmid containing a protein transduction domain (PTD) and a human A20 (hA20) gene fragment; the fusion protein was obtained by highly expressing this plasmid in the yeast $P i-$ chia pastoris GS115. The plasmid was obtained by adding 9xArg and EcoRI recognition sites to the end of the primer, and 6xHis-Tag and NotI recognition sites to its end. After sequencing, the hA20 gene fragment was inserted into plasmid pPIC9k to construct expression vector pPIC9k-PTD-hA20; then, we transfected GS115 with the vector and induced PTD-hA20 protein expression. We purified protein from the yeast fermentation supernatant using a nickel column. Human umbilical vein
\end{abstract}


endothelial cells (HUVECs) were cultured in high glucose medium (30 $\mathrm{mM}$ glucose) and in high glucose medium containing different concentrations of protein. Apoptosis of HUVECs was assayed by TUNEL 72 $\mathrm{h}$ later. The biological activity tests indicated that the fusion protein not only passed through the cell membrane freely, but also inhibited apoptosis of HUVECs induced by high glucose levels. We conclude that the fusion protein PTD-hA20 has potential for clinical use.

Key words: Protein transduction domain; hA20; Fusion protein; HUVECs; High glucose; Apoptosis

\section{INTRODUCTION}

Gene knockout experiments show that A20 is an important protective gene for endothelial cells. It not only inhibits endothelial cell injury and apoptosis caused by a variety of factors (such as trauma, infection, ischemia reperfusion, low-density lipoprotein, etc.), but also prevents monocyte recruitment and adhesion on the endothelial surface of blood vessels, and inhibits intimal hyperplasia induced by smooth muscle cell proliferation and migration, thus suppressing atherosclerosis (Patel et al., 2006; Wang et al., 2007; Lutz et al., 2008; Zeng et al., 2009). Our preliminary results showed that A20 overexpression not only protects human umbilical vein endothelial cells (HUVECs) from high glucose-induced injury, but also inhibits atherosclerosis in tissue-engineered blood vessels and prevents stent restenosis in blood vessels (Zhu et al., 2009; Zhou et al., 2009; Hou et al., 2011).

Given the important protective role of A20, it is essential to increase its level in cells effectively. Synthetic protein transduction peptides have increasingly attracted attention with the development of protein transduction technology (Tezgel et al., 2011; Papadopoulou et al., 2011). The protein transduction domain (PTD) composed of poly-L-arginine nonamer not only spontaneously crosses the cell membrane, but also delivers the target protein mainly to the cytoplasm (Schwarze et al., 1999; Hiroshi et al., 2006). Our target protein hA20 is a kind of cytoplasmic protein. Therefore, in this study, we designed, synthesized and expressed the fusion protein PTD-hA20 in vitro, which not only enters the cell by spontaneously crossing the cell membrane, but also inhibits high glucose-induced endothelial cell apoptosis.

\section{MATERIAL AND METHODS}

\section{Plasmids, strains and reagents}

Pichia pastoris GS115 and plasmid pPIC9k were donated by Professor Rao (Department of Microbiology, Third Military Medical University). The plasmid pCAGGSEHA20 and Escherichia coli DH5 $\alpha$ were preserved in our laboratory. A plasmid extraction kit, PCR kit and a variety of enzymes were purchased from Takara Corp. (Dalian, Liaoning, China). Anti-His-Tag monoclonal antibody was from Novagen Corp. Horseradish peroxidase (HRP)labeled goat anti-mouse antibody and rhodamine-labeled goat anti-rabbit $\operatorname{IgG}(\mathrm{H}+\mathrm{L})$ were purchased from Beijing Zhongshan Golden Bridge Biotechnology Co., Ltd. (China). G418 and POD apoptosis kits were obtained from Sigma. 


\section{Construction and identification of the recombinant plasmid}

The target gene fragment was obtained by PCR using pCAGGSEHA20 plasmid as the template. Sense primer: 5'-CCGGAATTCAGAAGACGTAGACGTAGAAGACGTAGAATG GCTGAACAAGTCC-3' and antisense primer: 5'-ATAAGAATGCGGCCGCTTAGTGGTG ATGGTGATGGTGGCCATACATCTGCTTG-3' were synthesized by Shanghai Biotech Co. (China). We constructed the expression vector pPIC9k-PTD-hA20 by inserting the amplified target gene fragment into the vector pPIC9k. The recombinant plasmid was confirmed by digestion and sequencing (Shanghai Biotech Co.).

\section{Plasmid pPIC9k-PTD-hA20 was transformed into GS115}

P. pastoris GS115 was cultured and transformed according to Invitrogen protocols. Electricity conversion conditions were as follows: electric rotor was $2 \mathrm{~mm}$, voltage $1.5 \mathrm{~V}$, capacitor $25 \mu \mathrm{F}$, resistance $200 \Omega$, and shock time $4.6 \mathrm{~ms}$. The control group was transfected with empty vector pPIC9k. The suspension was evenly coated on MD plates (each 200-600 $\mu \mathrm{L}$ coating a tablet) after the power switch. The yeast genome was extracted according to manufacturer instructions and was then used as a template for PCR amplification with the same primers and procedures as above.

\section{Screening of high protein expression strains and expression of fusion protein}

Each single colony of GS115 was screened by G418. The concentration gradient of G418 consisted of $0.25,0.5,0.75,1.0,1.5,1.75,2.0,3.0$, and $4.0 \mathrm{mg} / \mathrm{mL}$. We selected the high-expression strains when the concentration of G418 reached $4.0 \mathrm{mg} / \mathrm{mL}$.

A single colony of high-expression strains was inoculated into $25 \mathrm{~mL}$ medium (MGY), and the culture incubated with shaking at $250-300 \mathrm{rpm}$ and at $28^{\circ}-30^{\circ} \mathrm{C} . \mathrm{OD}_{600}$ was about $2-6$ $10 \mathrm{~h}$ later. GS115 were collected after centrifugation for $5 \mathrm{~min}$ at room temperature and resuspended in medium (BMMY) until $\mathrm{OD}_{600}=1.0$. The cells were then grown under the same conditions $\left(250-300 \mathrm{rpm}, 28^{\circ}-30^{\circ} \mathrm{C}\right)$; meanwhile, the methanol concentration was adjusted to $0.5 \%$ by adding methanol every $24 \mathrm{~h}$, and $0.1 \mathrm{mM} \mathrm{ZnCl}_{2}$ was also added to BMMY. The level of protein expression was analyzed at $0,6,12,24,36,48,60,72,84$, and $96 \mathrm{~h}$.

\section{Identification and purification of the recombinant protein}

As hA20 protein is a secreted protein, we extracted the target protein PTD-hA20 from the supernatant. The recombinant protein was identified by Western blotting. The first antibody was anti-His-Tag monoclonal antibody (Sigma) and the second antibody was HRPlabeled goat anti-mouse IgG $(\mathrm{H}+\mathrm{L})$ (Boster Corp., Wuhan, China). Six-histidine His-Tags had been added to the 3 '-end of the primer to facilitate the identification and purification of the protein. The results were analyzed with a gel imaging system.

To ensure that experimental results would not be marred by nonspecific proteins, the recombinant protein was purified through an Ni-NTA column. Proteins were separated with an eluent containing imidazole at different concentrations. The procedures were conducted under non-denaturing conditions. The concentration of the recombinant protein was determined using the Bradford method. 


\section{Isolation and culture of HUVECs}

HUVECs were prepared and cultured as previously described (Wang et al., 2007; Hou et al., 2011). The cells were identified as positive for von Willebrand factor (vWF) expression. The first antibody was rabbit anti-human vWF (Boster Corp.), and the second antibody was rhodamine-labeled fluorescent secondary antibody (Boster Corp.).

\section{Penetrating effect of the recombinant protein}

To test the penetrating effect of the recombinant proteins they were added to the medium, and total cytoplasmic protein was extracted and identified at 1,2,3, 4, and $5 \mathrm{~h}$. The specific protein was confirmed by Western blotting. The first antibody was anti-His-Tag monoclonal antibody, and the second antibody was HRP goat anti-mouse $\operatorname{IgG}(\mathrm{H}+\mathrm{L})($ Boster Corp.).

\section{Detection of biological functions of the recombinant protein}

The rate of HUVEC apoptosis induced by high glucose was detected using the TUNEL method. When the cells reached $60-70 \%$ confluence, the normal medium was replaced with high glucose medium (30 mM glucose) or high-glucose medium containing different concentrations of recombinant proteins (dilutions of 1:500, 1:400, 1:300, 1:200, and 1:100 of the primary concentration, $80 \mu \mathrm{g} / \mathrm{mL}$ ). The apoptosis rate of HUVECs was determined after $72 \mathrm{~h}$. The detailed steps are given in a previous report (Hou et al., 2011).

\section{Statistical analysis}

The experimental data were analyzed by SPSS 18.0, and the results are reported as means \pm SEM. Statistical significance of the differences between the two groups was determined by the Student $t$-test and $\mathrm{P}<0.01$ was considered to be statistically significant. Each experiment was repeated at least three times.

\section{RESULTS}

\section{Identification of recombinant plasmid pPIC9k-PTD-hA20}

We obtained the gene fragment (2447 bp) of PTD-hA20 by PCR. The fragment was verified by gel electrophoresis (Figure 1). We constructed the recombinant plasmid pPIC9KPTD-hA20, after inserting PTD-hA20 into vector pPIC9k (Figure 2). pPIC9k-PTD-hA20 was conversely identified by restriction enzyme digestion, and was confirmed by gel electrophoresis (Figure 3). In addition, the sequence of the fragment determined was consistent with that of the template and that reported by GenBank.

\section{Insertion of PTD-hA20 into the yeast genome}

After being linearized, pPIC9k-PTD-hA20 was transformed into the yeast GS115. 
The extracted yeast genome was used as a template for PCR. The results showed that pPIC9kPTD-hA20 successfully integrated with the yeast genome (Figure 4).

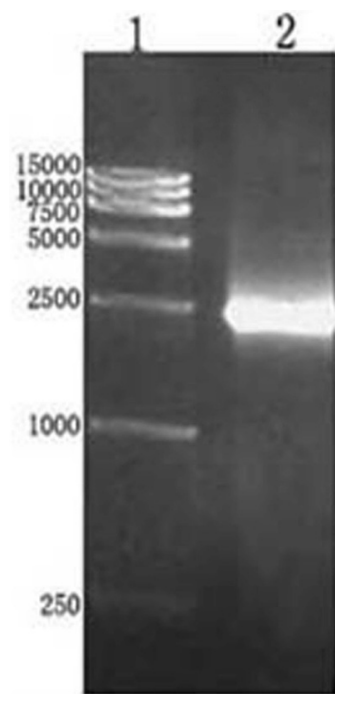

Figure 1. Result of the polymerase chain reaction. Lane $1=$ DNA marker; lane $2=$ PTD-hA20.

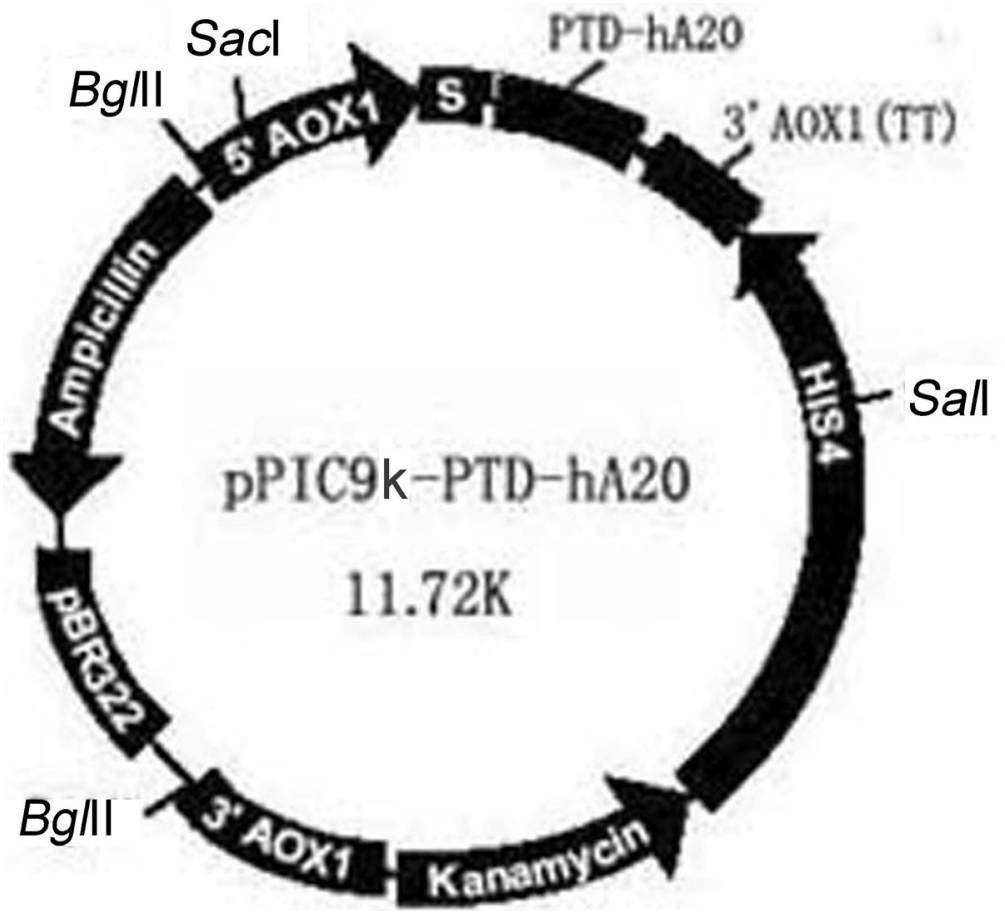

Figure 2. Map of pPIC9k-PTD-hA20. 


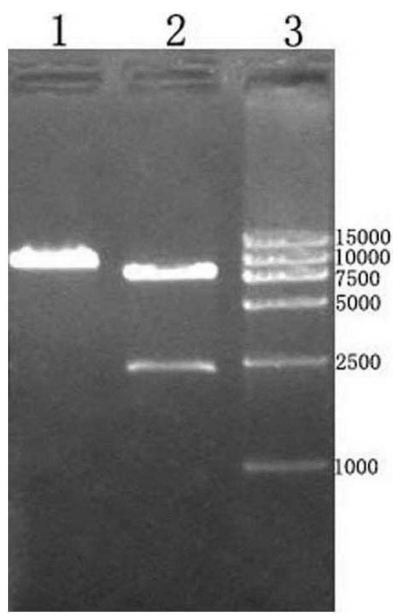

Figure 3. Digestion results of pPIC9k-PTD-hA20. Lane 1 = Digested by EcoRI; lane 2 = digested by EcoRI and NotI; lane 3 = DNA marker.

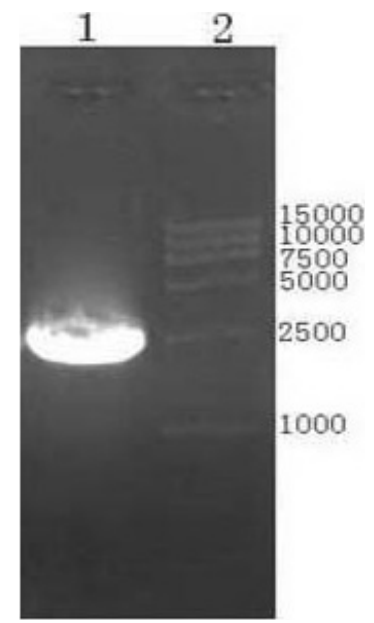

Figure 4. Result of the polymerase chain reaction. Lane $1=$ PTD-hA20; lane 2 = DNA marker.

\section{Identification of the recombinant protein}

The molecular mass of the recombinant protein was $\sim 92 \mathrm{kDa}$. Gel electrophoresis showed that a clear band existed between 66.4 and $97.2 \mathrm{kDa}$ (Figure 5A). Western blotting further confirmed that the band was the target protein (Figure 5B).

\section{Protein purification}

Gel electrophoresis showed that the vast majority of nonspecific proteins were removed, indicating that the recombinant protein had been purified (Figure 6). 


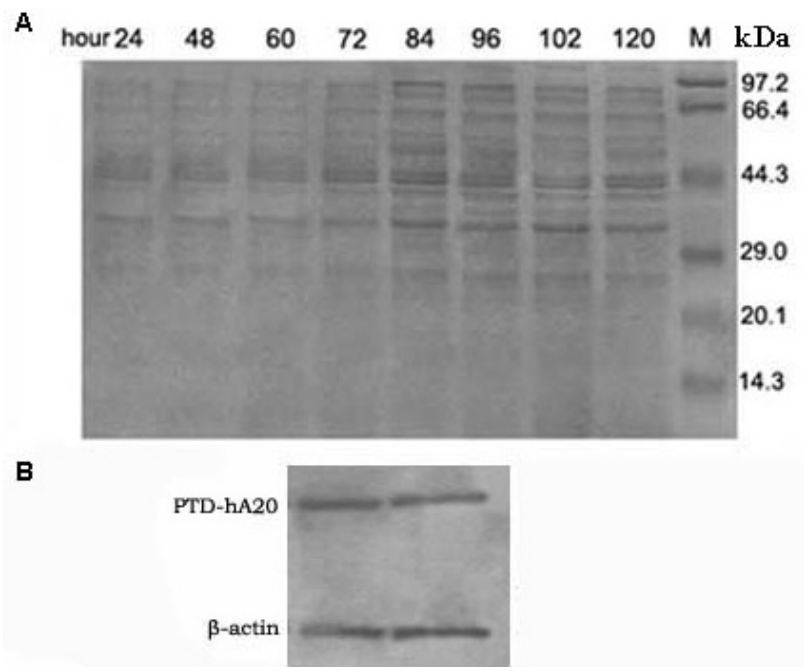

Figure 5. Results of gel electrophoresis and Western blot. A. Gel electrophoresis results of yeast supernatant at different times. B. Results of Western blot.

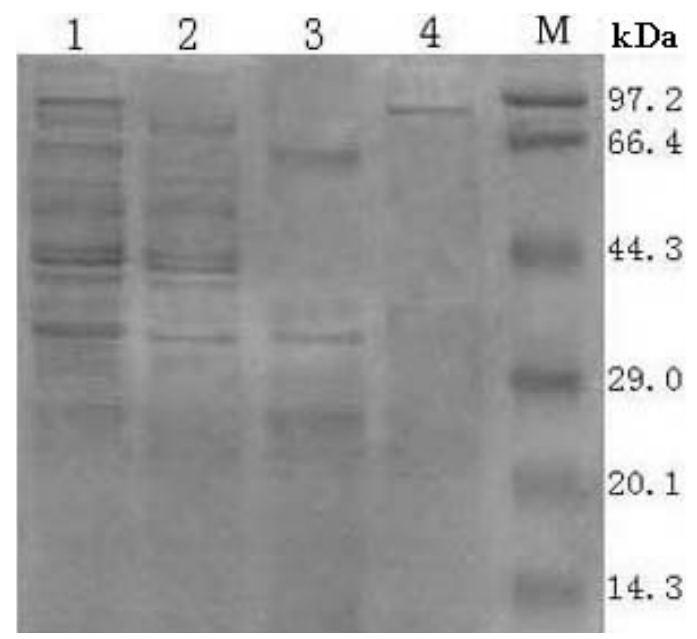

Figure 6. Depuration results of recombinant protein. Lane $1=$ Supernatant of yeast; lane 2 = protein penetration peak; lane 3 = eluting peak when iminazole is $20 \mathrm{mM}$; lane $4=$ eluting peak when iminazole is $250 \mathrm{mM}$; lane $M=$ protein molecular weight marker.

\section{Penetrating effect of the protein}

Western blot analysis of the total cytoplasmic protein showed that recombinant protein could pass through the membrane into the cytoplasm in $1 \mathrm{~h}$, indicating that the PTD had good activity and could successfully transport the target protein into the cell. Immunohistochemical results showed that the protein was mainly localized in the cytoplasm (Figure 7). 
A

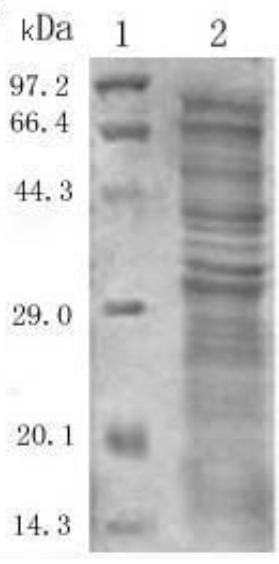

B

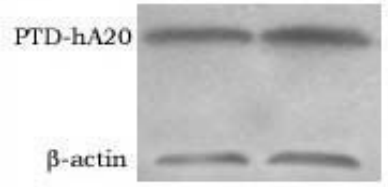

C

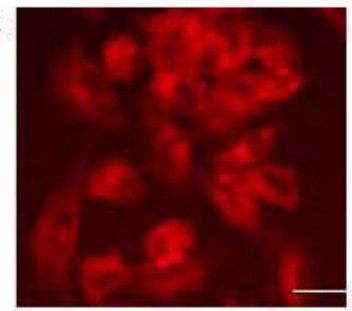

Figure 7. Identification results of recombinant protein. A. Gel electrophoresis results of cytoplasm protein. Lane $1=$ Protein marker; lane $2=$ total cytoplasmic protein. B. Map of Western blot. C. Immunohistochemical result. Scale bar $=25 \mu \mathrm{m}$.

\section{Detection of apoptosis}

It can be seen from the Figure 8 that the recombinant protein protected endothelial cells against injury induced by high-concentration glucose $(\mathrm{P}<0.01)$. In a certain concentration range, the rate of cell apoptosis was negatively correlated with protein concentration: when the ratio (protein/medium) was 1:200, the rate of cell apoptosis reached a minimum value and it means that the protective effect of the A20 protein is the most notable. Then, even if we increase the concentration of protein, there is almost no change in the rate of cell apoptosis.

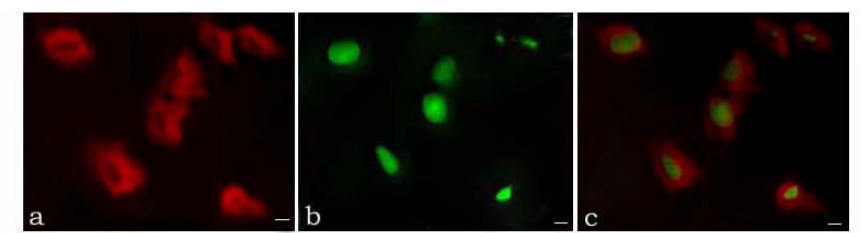

d

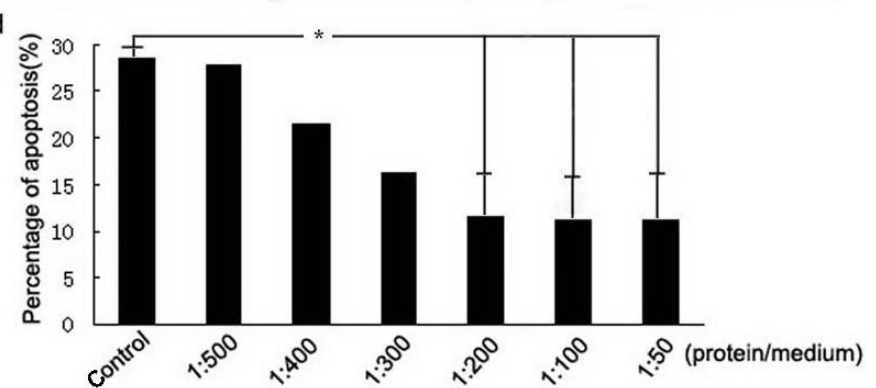

Figure 8. Apoptosis of human umbilical vein endothelial cells (HUVECs) was determined by fluorescence microscope (400X). a. HUVECs were determined by von Willebrand factor. b. Apoptosis of HUVECs in the same scope was counted by fluorescence microscope. $\mathbf{c} . \mathrm{a}+\mathrm{b}$. $\mathbf{d}$. Histogram of cell apoptosis rate. ${ }^{*} \mathrm{P}<0.01$ compared with the control group. Scale bar $=25 \mu \mathrm{m}$. 


\section{DISCUSSION}

A20 has long been the focus of attention because of its important protective role in endothelial cells. However, the vast majority of previous studies on A20 have mainly focused on the genetic level, such as the transduction of foreign genes into cells or animals by liposomes or virus particles. However, its practical application is limited by many factors, such as poor operability, low transfer efficiency, poor stability, biological toxicity, etc. Therefore, at present, studies on the A20 gene are limited to cells or animals. It is imperative for us to further explore its clinical value. Therefore, we hypothesized that if protein hA20 can be obtained in vitro and can spontaneously cross the cell membrane, its study will be more convenient. As a result, its application will be greatly expanded, and it is likely to be used in clinical practice.

We all know that, under normal physiological conditions, only a few small molecules can pass through eukaryotic cell membranes, while proteins and other biological macromolecules are usually transported by endocytosis or exocytosis. It is very difficult for exogenous peptides and other biological macromolecules to enter cells and treat diseases. However, with the development of protein transduction technology, many genes with beneficial effects have been cloned, expressed, and transported to the human body by various means. Mitchell et al. (2000) and Wender et al. (2000) synthesized a short peptide with nine L-arginines (R9) or nine D-arginines (r9), respectively, with a transduction efficiency 20-100 times that of Tat-PTD. Mitsui et al. (2006) found that the target protein carried by the PTD protein was mainly located in the cytoplasm, where our target protein hA20 was found.

In this study, we amplified the human A20 gene by PCR. We successfully constructed the plasmid pPIC9k-PTD-hA20 and added poly-L-arginine (nonamer) with a high protein transduction efficiency to its 5 '-end. We then obtained the reconstructed protein PTD-hA20 through expression in yeast. Not only could the fusion protein cross the membrane to enter cells, but it was mainly located in the cytoplasm. Further functional experiments confirmed that the protein has good biological activity and can significantly inhibit high glucose-induced endothelial cell apoptosis. Our study provides a new method for future research on A20 and offers a novel therapy for diabetic complications.

\section{ACKNOWLEDGMENTS}

Research supported by the National Science Foundation of China (\#31100707) and the Natural Science Foundation of Chongqing (\#CSTC, \#2010BB5176). We would like to thank Ms. Xie-Wan Chen and Ms. Xiao-Qing Zhan (Medical English Department, Third Military Medical University, China) for a critical reading of the manuscript and kindly giving precious advice.

\section{REFERENCES}

Hiroshi M, Takashi I, Reilo K, Shibagaki N, et al. (2006). Polyarginine-mediated protein delivery to dendritic cells presents antigen more efficiently onto MHC class I and class II and elicits superior antitumor immunity. J. Investig. Dermatol. 126: 1804-1812.

Hou CL, Zhang W, Wei Y, Mi JH, et al. (2011). Zinc finger protein A20 overexpression inhibits monocyte homing and protects endothelial cells from injury induced by high glucose. Genet. Mol. Res. 10: 1050-1059.

Lutz J, Luong 1A, Strobl M, Deng M, et al. (2008). The A20 gene protects kidneys from ischaemia/reperfusion injury by suppressing pro-inflammatory activation. J. Mol. Med. 86: 1329-1339.

Mitchell DJ, Kim DT, Steinman L, Fathman CG, et al. (2000). Polyarginine enters cells more efficiently than other 
polycationic homopolymers. J. Pept. Res. 56: 318-325.

Mitsui H, Inozume T, Kitamura R, Shibagaki N, et al. (2006). Polyarginine-mediated protein delivery to dendritic cells presents antigen more efficiently onto MHC class I and class II and elicits superior antitumor immunity. J. Invest Dermatol. 126: 1804-1812.

Papadopoulou LC and Tsiftsoglou AS (2011). Transduction of human recombinant proteins into mitochondria as a protein therapeutic approach for mitochondrial disorders. Pharm. Res. 28: 2639-2656.

Patel VI, Daniel S, Longo CR, Shrikhande GV, et al. (2006). A20, a modulator of smooth muscle cell proliferation and apoptosis, prevents and induces regression of neointimal hyperplasia. FASEB J. 20: 1418-1430.

Schwarze SR, Ho A, Vocero-Akbani A and Dowdy SF (1999). In vivo protein transduction: delivery of a biologically active protein into the mouse. Science 285: 1569-1572.

Tezgel AO, Telfer JC and Tew GN (2011). De novo designed protein transduction domain mimics from simple synthetic polymers. Biomacromolecules 12: 3078-3083.

Wang AB, Li HL, Zhang R, She ZG, et al. (2007). A20 attenuates vascular smooth muscle cell proliferation and migration through blocking PI3k/Akt singling in vitro and in vivo. J. Biomed. Sci. 14: 357-371.

Wender PA, Mitchell DJ, Pattabiraman K, Pelkey ET, et al. (2000). The design, synthesis, and evaluation of molecules that enable or enhance cellular uptake: peptoid molecular transporters. Proc. Natl. Acad. Sci. U. S. A. 97: 13003-13008.

Zeng W, Li L, Yuan W, Wei Y, et al. (2009). A20 overexpression inhibits low shear flow-induced CD14-positive monocyte recruitment to endothelial cells. Biorheology 46: 21-30.

Zhou Z, Shi S, Song M, Huang H, et al. (2009). Development of transgenic endothelial progenitor cell-seeded stents. $J$. Biomed. Mater. Res. A 91: 623-628.

Zhu C, Ying D, Mi J, Li L, et al. (2008). Development of anti-atherosclerotic tissue-engineered blood vessel by A20regulated endothelial progenitor cells seeding decellularized vascular matrix. Biomaterials 29: 2628-2636. 